\title{
ZUR HERKUNFT VON LEUGA*
}

Summary: According to the communis opinio, Lat. leuga was a Gaulish loanword, survived in the Romance languages and was borrowed into Old English. However, this scenario faces three unsolved problems: the non-Celtic diphthong -eu-, the Proto-Romance form *legua and the fact that the Old English word cannot continue the Latin form on phonological grounds. This paper argues that all these problems can regularly be solved by the reconstructed West Germanic and Gothic cognates of the Old English word borrowed into Gaulish and early Romance dialects, respectively.

Key words: Latin etymology, Romance etymology, Gaulish, Germanic, West Germanic, Old English

\section{STAND DER FORSCHUNG}

Der Forschungsstand der Etymologie des lat. Wortes leuga, eine Längenmaßeinheit von 1500 Schritten' besteht im Grunde aus den folgenden drei Aussagen:

1) Leuga ist ein keltisches, genauer gesagt ein gallisches Lehnwort im Lateinischen, ${ }^{1}$

* Dieser Aufsatz ist im Rahmen des Forschungsprojekts „Lendület (Momentum) Research Group for Computational Latin Dialectology“ zustande gekommen. Ich bedanke mich herzlich bei Anja Busse für die Korrektur meines Deutsch.

${ }^{1}$ Bosworth, J. - TOlLer, T. N.: An Anglo-Saxon Dictionary I-II. Oxford 1882-1898, 635; BeSNIER, M.: Notes sur les routes de la Gaule romaine. REA 31 (1929) 334-338, hier 336; MEYER-LÜBKE, W.: Romanisches etymologisches Wörterbuch. Heidelberg $1935^{3}$, 810; INSTINSKY, H. U.: Septimius Severus und der Ausbau des raetischen Straßennetzes. Klio 31 (1938) 33-50, hier 48; ERNOUT, A. - MEILLET, A.: Dictionnaire étymologique de la langue latine. Histoire des mots. Paris $1951^{3}$, 628; WALDE, A. - HOFMANN, J. B.: Lateinisches etymologisches Wörterbuch. Heidelberg 1938-1954 ${ }^{3}, 787$; VON WARTBURG, W.: Französisches Etymologisches Wörterbuch. Eine darstellung des galloromanischen sprachschatzes 5. Basel 1950, 262; SCHMIDT, K. H.: Keltisches Wortgut im Lateinischen. Glotta 44 (1966) 151-174, hier 168; Oxford Latin Dictionary. Oxford 1968, 1019; Thesaurus Linguae Latinae 7.2. Leipzig 1966-1979, 1196; MACHADO, J. P.: Dicionário etimológico da língua portuguesa. Com a mais antiga documentação escrita e conhecida de muitos dos vocábulos estudados 3. Lisboa 1977³, 399 (implizit); COROMINAS, J.: 
was schon von antiken Autoren so angenommen wurde (Grom. p. 373. 17; Hier. in Ioel 3. 18; Iord. Get. 192; Isid. Orig. 15. 16. 1; Gloss. V 217. 8). ${ }^{2}$

2) Leuga lebt weiter in den romanischen Sprachen: in frz. lieue, altprov. lega (entlehnt ins Italische als lega) und legua, kat. llegua, sp. legua, port. légua, ${ }^{3}$ und wurde schließlich auch ins Englische als league entlehnt. ${ }^{4}$

3) Leuga wurde als lēowe ,Meile“ außerdem auch ins Angelsächsische entlehnt. ${ }^{5}$ Wie die Literaturangaben zeigen, herrscht praktisch seit Anfang der Forschung Einigkeit darüber, dass diese Aussagen korrekt sind.

\section{DIE PROBLEME}

Es gibt allerdings drei Probleme, die durch genannte Behauptungen nicht erklärt werden:

1. Leuga zeigt einen eu-Diphthong, -eu- existiert allerdings im Keltischen wegen des urkeltischen Lautwandels *eu $>$ ou eigentlich nicht (zu den angeblichen Ausnahmen s. unten). ${ }^{6}$

2. Die meisten romanischen Fortsetzer zeigen keine Urform leuga, sondern *legua, die einzige Ausnahme ist altkat. und dial. kat. lleuga. ${ }^{7}$

Diccionario crítico etimológico castellano e hispánico. 3: G-Ma. Madrid 1980, 625; PORZIO GERNIA, M. L.: Gli elementi celtici del latino. In CAMPANILE, E. (Hg.): I Celti d'Italia. Pisa 1981, 97-122, hier 111; Cortelazzo, M. - Zolli, P.: Dizionario etimologico della lingua italiana. Bologna 1999 ${ }^{2}$, 859; LAMBERT, P.-Y.: La langue gauloise. Description linguistique, commentaire d'inscriptions choisies. Paris 2003 2 , 196; ADAMS, J. N.: The Regional Diversification of Latin, 200 BC-AD 600. Cambridge 2007, 355; WeISS, M.: Outline of the Historical and Comparative Grammar of Latin. Ann Arbor - New York $2009,487$.

${ }^{2}$ Vgl. Holder, A.: Alt-celtischer Sprachschatz. 2: I-T. Leipzig 1904, 198; ThLL (Anm. 1) 1196.

${ }^{3}$ MeYER-LÜBKE (Anm. 1) 810; vON WARTBURG (Anm. 1) 262; ThLL (Anm. 1) 1196; MACHADO (Anm. 1) 399; COROMINAS (Anm. 1) 625; CORTELAZZO - ZOLLI (Anm. 1) 859; vgl. auch HoldER (Anm. 2) 197; ADAMS (Anm. 1) 355; WeISS (Anm. 1) 487 Anm. 48 (seine Behauptung, die spanische Form sei aus dem Altprovenzalischen entlehnt, muss ein Tippfehler sein, vgl. COROMINAS (Anm. 1) 625).

${ }^{4}$ Oxford English Dictionary. s.v. www.oed.com (letzter Zugriff: 20. Dezember 2018).

${ }^{5}$ Bosworth-Toller (Anm. 1) 635; Holder (Anm. 2) 197; WALDE-HofMANN (Anm. 1) 787 (,wohl“); Whatmough, J.: The Dialects of Ancient Gaul. Prolegomena and Records of the Dialects. Cambridge, Mass. 1970, 572; COROMINAS (Anm. 1) 625 (implizit). Das neue Wörterbuch des Altenglischen ist momentan nur bis zum Buchstaben I verfügbar (DIPAOLO HEALEY, A. ET AL. [Hg.]: Dictionary of Old English. https://www.doe.utoronto.ca [letzter Zugriff: 2. Dezember 2018]).

${ }^{6}$ MCCone, K.: Towards a Relative Chronology of Ancient and Medieval Celtic Sound Change. Maynooth 1996, 64; LAMBERT (Anm. 1) 42; Sims-Williams, P.: The Celtic Inscriptions of Britain. Phonology and Chronology, c. 400-1200. Oxford-Malden 2003, 23; SCHUMACHER, S.: Die keltischen Primärverben. Ein vergleichendes, etymologisches und morphologisches Lexikon. Innsbruck 2004, 116, 122 [auch wenn seine Formulierung uneindeutig ist: ,urkelt. *eu, später *ou“]; MATASOVIĆ, R.: Etymological Dictionary of Proto-Celtic. Leiden-Boston 2009, 9; STIFTER, D.: The Phonology of Celtic. In KleIN, J. - JosePH, B. - FrITZ, M. (Hg.): Handbook of Comparative and Historical Indo-European Linguistics 2. Berlin-Boston 2017, 1188-1202, hier 1196-1198.

${ }^{7}$ COROMinas (Anm. 1) 625 mit Lit.; CORTElaZZO-Zolli (Anm. 1) 859; vgl. schon MeYer, W.: Labialisierung von Gutturalen im Nordfranzösischen. ZRPh 11 (1888) 538-542, hier 539, auch vON WARTBURG (Anm. 1) 262; TLL (Anm. 1) 1196. Einige Forscher (HoLDER [Anm. 1] 197; ThLL [Anm. 1] 1196; WEISS [Anm. 1] 487 Anm. 48) zitieren noch altprov. leuga, das jedoch nach dem neuesten altokzi- 
3. Das angelsächsische Wort lēowe kann keine Entlehnung darstellen, weder aus einer keltischen Sprache (trotz der Annahme Holthausens aus kelt. leuca, ${ }^{8}$ zur Form s. unten) noch aus dem Lateinischen selbst, weil diese Form aus phonologischen Gründen nicht aus leuga stammen kann (vgl. z.B. ags. flēogan ,fliegen' < urgerm. *fleugana). ${ }^{9}$

Im Folgenden wird diesen Fragen nachgegangen, um schließlich eine Erklärung dafür anbieten zu können.

\subsection{Der eu-Diphthong}

Das Problem des eu-Diphthongs wurde schon von Delamarre und Weiss beobachtet. ${ }^{10}$ Beide schlagen vor, dass es sich um eine sekundäre Form handelt. Ohne eine Erklärung für die Entstehung eines solchen sekundären Diphthongs bleibt diese Annahme allerdings willkürlich. Es gibt tatsächlich einige isolierte keltische Wörter, die einen solchen Diphthong aufweisen und über deren Erklärung noch keine Einigkeit herrscht: Einige Forscher glauben, dass der Wandel *eu $>*$ ou keinen urkeltischen, sondern einen späteren, einzelsprachlichen (d.h. in diesem Fall gallischen) Lautwandel darstellt. ${ }^{11}$ McCone macht dagegen darauf aufmerksam, dass die Formen mit -eu- keine archaischen Wörter sein können, weil sie zunächst in lateinischen Texten erscheinen oder in gallischen Inschriften, die mit lateinischen Buchstaben geschrieben wurden, somit also später als die Formen mit -ou-. ${ }^{12}$ Er erwägt zwei Möglichkeiten: einen sporadischen gallischen Lautwandel ou > eu oder (und diese Lösung

tanischen Wörterbuch (STEMPEL, W.-D. - SELIG, M. [Hg.]: Dictionnaire de l'Occitan Médiéval. München, http://www.dom-en-ligne.de s.v. lega [letzter Zugriff: 27. August 2018]) nicht belegt ist.

${ }^{8}$ Holthausen, F.: Altenglisches etymologisches Wörterbuch. Heidelberg 1934, 200.

${ }^{9}$ Zur angelsächsischen Lautgeschichte im Allgemeinen s. z.B. RINGE, D. - TAYLOR, A.: The Development of Old English. Oxford 2014, bes. 174, $309 \mathrm{zu}$ diesem Beispiel.

${ }^{10}$ DelamarRe, X.: Dictionnaire de la langue gauloise. Une approche linguistique du vieux-celtique continental. Paris $2003^{2}, 200$ und WEISS (Anm. 1) 487 Anm. 38.

${ }^{11}$ PEDERSEN, H.: Vergleichende Grammatik der keltischen Sprachen I. Einleitung und Lautlehre. Göttingen 1909, 53-54; DotTIN, G.: La langue gauloise. Grammaire, textes et glossaire. Paris 1920, 97; THURNEYSEN, R.: Keltisches. ZVS 59 (1932) 1-18, hier 15-16; VON KIENLE, R.: Italiker und Kelten. Wörter und Sachen 17 (1936) 98-153, hier 103; JACKSON, K.: Language and History in Early Britain. A Chronological Survey of the Brittonic Languages. First to Twelfth Century A.D. Edinburgh 1953, 305; WATKIns, C.: The Phonemics of Gaulish: The Dialect of Narbonensis. Language 31 (1955) 9-19, hier 13 (aber vgl. unten); SCHMIDT, K. H.: Die Komposition in gallischen Personennamen. Zeitschrift für celtische Philologie 26 (1957) 33-301, hier 277; Evans, D. E.: Gaulish Personal Names. A Study of Some Continental Celtic Formations. Oxford 1967, 396; SCHRIJVER, P.: Studies in British Celtic Historical Phonology. Amsterdam-Atlanta 1995, 192, 194, 327; ESKA, J. F.: Continental Celtic. In WoODARD, R. D. (Hg.): The Cambridge Encyclopedia of the World's Ancient Languages. Cambridge 2004, 857-880, hier 863; ESKA, J. F. - Evans, D. E.: Continental Celtic. In BALL, M. J. - Müller, N. (Hg.): The Celtic Languages. London-New York 2009, 28-54, hier 40. Ähnlich SiMS-WiLliAMS, P.: Celtic. In KAPOVIĆ, M. (Hg.): The Indo-European Languages. Oxford-New York 2017, 352-386, hier 362, der von einer „early tendency, seen already in Lepontic and Gaulish“ spricht (früher ließ er die Frage offen: Common Celtic, Gallo-Brittonic and Insular Celtic. In LAmBerT, P.-Y. - PINAUlT, G.-J. (Hg.): Gaulois et Celtique Continental. Genève 2007, 309-353, hier 313).

${ }_{12}$ MCCONE (Anm. 6) 8, vgl. schon WATKIns (Anm. 11) 13. 
bevorzugt er) dass es sich nur um eine rein graphische Konvention handelt: das Lateinische verfügte über keinen ou-Diphthong, weshalb die Zeichensequenz $\langle$ eu $\rangle$ für den Diphthong [ou] verwendet wurde. ${ }^{13}$ Diese Möglichkeit kann man aber bestimmt ausschließen: abgesehen davon, dass lateinsprachige Autoren normalerweise keine Probleme hatten, ou-haltige fremde Namen mit -ou- zu schreiben (vgl. z.B. Boudicca bei Tacitus, $A g$. 16), wurden die gallischen Inschriften, in denen -eu- erscheint, auf Gallisch verfasst, weshalb es irrelevant ist, ob das Lateinische über einen eu-Diphthong verfügte. Weshalb hätten die Gallier die Buchstaben $\langle$ eu $\rangle$ statt $\langle o u\rangle$ für ihren Diphthong $\langle\mathrm{ou}\rangle$ verwenden sollen?

Unabhängig davon besteht kein Zweifel daran, dass die Schreibung 〈eu〉 in leuga eine Lautfolge mit [eu] wiedergibt. Dies zeigen sowohl die eindeutigen römischen Inschriften und Texte der Autoren als auch die romanische Fortsetzung sowie die griechische Umschrift bei Hesychios (eine Glosse zur Sprache der Galater: $\lambda \varepsilon v ́ \gamma \eta$ •

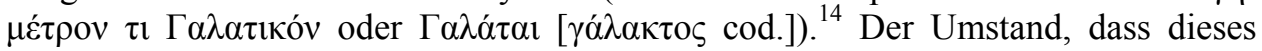
Wort keinen Lautwandel *eu > ou zeigt, kann mit drei verschiedenen Szenarien erklärt werden:

Erstens: es handelt sich um einen Archaismus, der aber bei einem gebräuchlichen Wort wie ,Meile‘ sehr fragwürdig ist.

Zweitens: es handelt sich um das Ergebnis eines späten, sporadischen gallischen Lautwandels aus *louga. Dagegen spricht allerdings die galatische Form, die zeigt, dass leuga schon spätestens im 3. Jh. v. Chr. (d.h. zur Zeit der Einwanderung der Galater in Kleinasien) leuga lautete, weshalb es mit einem späten *ou > eu Lautwandel nicht erklärt werden kann.

Drittens: es handelt sich um eine Entlehnung nach dem Lautwandel *eu $>* o u$, was aus phonologischer Sicht einwandfrei und einleuchtend ist.

${ }^{13}$ McCone folgen auch MATASOvić (Anm. 6) 15 und STIFTER (Anm. 6) 1197.

${ }^{14}$ Vgl. Holder (Anm. 1) 198; WeISGERBER, L.: Galatische Sprachreste. In HelM, R. ET AL.: Natalicium. Johannes Geffcken zum 70. Geburtstag. 2. Mai 1931. Gewidmet von Freunden, Kollegen und Schülern. Heidelberg 1931, 151-175, hier 164; BOLELLI, T.: Le voci di origine gallica del Romanisches Etymologisches Wörterbuch di W. Meyer-Lübke 2. L'Italia dialettale 18 (1942) 33-74, hier 50; WHATMOUGH (Anm. 5) 572; BILly, P.-H.: Thesaurus Linguae Gallicae. Hildesheim-Zürich 1993, 94. FREEMAN, PH.: The Galatian Language - A Comprehensive Survey of the Language of the Ancient Celts in Greco-Roman Asia Minor. Lewiston-Queenston-Lampeter 2001, 6-7 behauptet, eine galatische Herkunft dieses Wortes lasse sich nicht nachweisen, weil sich der Begriff Galatai bei den griechischsprachigen Autoren auf alle Kelten beziehen konnte. In Anbetracht seiner Herkunft und Quellen ist es allerdings kaum wahrscheinlich, dass Hesychios gallische und nicht galatische Wörter aufzeichnete. Zwar könnte man dieses Wort für eine Entlehnung aus dem Lateinischen halten (in Zusammenhang mit der lateinsprachigen östlichen Reichsverwaltung und mit dem Erscheinen des Lateinischen in der östlichen Provinzverwaltung während der Dominatszeit, s. zusammenfassend ADAMIK, B.: Sprachpolitik im Römischen Reich. Zur Frage einer angenommenen sprachpolitischen Reform unter der Tetrarchie. AAntHung 50 (2010) 409418, hier 410-413 mit Lit.), dagegen spricht allerdings der Umstand, dass der Gebrauch dieses Wortes auf bestimmte westliche Provinzen (Aquitania, Gallia, Belgica und die beiden Germaniae) beschränkt war (VON WARTBURG [Anm. 1] 262; ADAMS [Anm. 1] 355). Das Wort ist jedenfalls nicht griechisch, wie OnIOns, C. T.: The Oxford Dictionary of English Etymology. Oxford 1966, 520 und BATTISTI, C. Alessio, G.: Dizionario etimologico italiano 3. Firenze 1952, 2193 glauben, und bedeutet nicht ,una misura di latte‘, was letztere aufgrund ihrer wortgetreuen Interpretation der Hesychiosstelle annehmen. 
Das wahrscheinlichste Szenario ist daher, dass leuga eine Entlehnung im Gallischen darstellt. Whatmough und Delamarre hatten bereits die Keltizität dieses Wortes in Zweifel gezogen (,not certain“ bzw. „très incertaine“) allerdings nur aus dem Grund, dass kein inselkeltischer Kognat belegt ist, ${ }^{15}$ obwohl diese Begründung natürlich nicht ausreicht. ${ }^{16}$ Um die Frage beantworten zu können, woher leuga sonst stammen könnte, müssen die beiden anderen Probleme besprochen werden.

\subsection{Die romanische Doppelform}

Wie die oben zitierte Liste zeigt, stammen fast alle romanischen Formen aus *legua, nicht aus dem belegten leuga. Diese Doppelform wurde bislang nicht erklärt. Meyers Annahme eines „Vorrückens labialer Affektion“"17 ist ad hoc, es gibt keine Motivation für eine Metathese (der Diphthong -eu- existiert im Lateinischen, z.B. seu, neu, $c e u$, auch wenn er nicht besonders häufig vorkommt). ${ }^{18}$ Ebenso ist die zweifache Lautsubstitution eines fremden Phonems nicht wahrscheinlich, weil ein Phonem $/ \mathrm{g}^{\mathrm{w}} /$, das man aus -ug- / -gu- erschließen könnte, auch dem Lateinischen wohl bekannt ist (lingua, sanguen, urgueo, usw.). Corominas schlägt vor, dass /g/ nach /w/ geschwunden sei und *lewa zu *legua wurde, wie im Falle von tregua, analog zur anlautenden $g$-Prothese bei germanischen Lehnwörtern (Typ guardar). ${ }^{19}$ Man kann allerdings die anlautende Position nicht als Basis für eine Erklärung heranziehen, weil nicht gesichert ist, dass diese Entwicklung mit der in der inlautenden Position identisch ist. Der Fall tregua zeigt sogar, dass der Vorschlag von Corominas nicht stichhaltig ist: it. trégua / trègua, prov. tregua, sp. tregua, port. trégua, altkat. tregua, altprov. trega gehen auf *tregua, Waffenstillstand' zurück, dagegen setzten fr. trève und kat. treva (mit altit. treva) *trewa fort. ${ }^{20}$ Bekanntlich setzt *tregua die gotische (triggwa, Bündnis, Bund') und *trewa die westgermanische Form *treuwa (vgl. z.B. ags. trēow, as.

${ }^{15}$ Whatmough (Anm. 5) 572 und Delamarre (Anm. 10) 200. Man beachte, dass die bretonische Form leo / lev ein Lehnwort aus dem Französischen darstellt (vgl. schon PEDERSEN [Anm. 11] 212 , ihm folgt VON WARTBURG [Anm. 1] 262; gegen DotTin [Anm. 11] 265 und SkEAT, W. W.: An Etymological Dictionary of the English Language. New Edition Revised and Enlarged. Oxford 1961, 333). Interessanterweise schlägt WHATMOUGH (Anm. 5) 572 vor, dass das angelsächsische Wort ,probably“ in Britannien entlehnt wurde, weil dieses Wort den anderen germanischen Sprachen fehlt. Dies würde allerdings bedeuten, dass es auch einen inselkeltischen Kognat gab, weshalb man nach den Kriterien Whatmoughs dieses Wort auch für das Urkeltische annehmen sollte. Diese Entlehnung ist allerdings lautlich nicht möglich, vgl. oben.

${ }^{16}$ Dieses Wort wurde auch im urkeltischen Wörterbuch von MATASOVIĆ (Anm. 6) außer Acht gelassen, dieses Wörterbuch ist allerdings bekanntlich unvollständig. Für überholte indogermanische Etymologien s. WALDE - HOFMANN (Anm. 1) 787.

${ }^{17}$ MeYER (Anm. 7) 359.

${ }^{18}$ Vgl. schon COROMINAS (Anm. 1) 625 contra RONJAT, J.: Grammaire Istorique des Parlers Provençaux Modernes 2.1. Fonétique 2. Consonnes et fénomènes généraux. Montpellier 1932, 247.

${ }^{19}$ COROMINAS (Anm. 1) 625 (vgl. schon MEYER-LÜBKE [Anm. 1] 742), seine Erklärung wurde in CORTELAZZO - ZOLLI (Anm. 1) 859 als ,,sehr kompliziert“ abgelehnt.

${ }^{20}$ Für die Formen s. MEYER-LÜBKE (Anm. 1) 742. 
treuwa, ahd. triuwa, Vereinbarung, Vertrag“) des gleichen germanischen Wortes *trewwō fort. ${ }^{21}$

Dementsprechend können die Formen, die *legua fortsetzen, gotischen Ursprungs sein: es überrascht nicht, dass genau diejenigen romanischen Sprachen diese Form fortsetzen, die sich im Gebiet des westgotischen Königreichs herausgebildet haben (Provenzalisch, Katalanisch, Spanisch und Portugiesisch). Es wäre allerdings sicherer, wenn man die Existenz eines solchen Wortes auch in den germanischen Sprachen nachweisen könnte. Dies ist in der Tat der Fall: wenn *legua aus got. *liggwa stammt, sollte der westgermanische Kognat *leuwa lauten. Dies entspricht genau der Form, auf die ags. lēowe zurückgeht bzw. zurückgehen kann. ${ }^{22}$

\subsection{Möglichkeiten zur Erklärung von leuga}

Wie ist leuga im Lichte des germanischen *leuwa / *liggwa zu beurteilen? Es gibt mehrere Möglichkeiten, wobei die Voraussetzung für eine akzeptable Lösung ist, dass dadurch sowohl der genaue Weg der Entlehnung als auch die Form leuga selbst erklärt werden können.

Theoretisch ist es möglich, dass leuga eigentlich kein gallisches sondern ein germanisches Wort ist, denn es wurde ja in Gallia und in Germania (neben Aquitania und Belgica) verwendet (vgl. oben). Dies würde allerdings der übereinstimmenden Aussage mehrerer, voneinander unabhängiger antiker Autoren widersprechen, was man damit erklären könnte, dass die sprachliche Einstufung dieser Autoren oft fehlerhaft war. Dies ist hier aber kaum wahrscheinlich: nicht nur wegen der Anzahl und Unabhängigkeit der Autoren, sondern vor allem auch weil einer der Autoren - Hieronymos - zumindest über Grundkenntnisse des Gallischen verfügt haben muss, da er bestimmen konnte, dass die Sprache der Galater mit der Sprache der Treverer in

${ }^{21}$ VON WARTBURG, W.: Französisches Etymologisches Wörterbuch. Eine darstellung des galloromanischen sprachschatzes 17. Basel 1966, 361; MACHADO, J. P.: Dicionário etimológico da língua portuguesa. Com a mais antiga documentação escrita e conhecida de muitos dos vocábulos estudados 5. Lisboa $1977^{3}$, 332; COROMINAS, J.: Diccionario crítico etimológico castellano e hispánico 5. Madrid 1983, 618; vgl. schon BRÜCH, J.: Der Einfluss der germanischen Sprachen auf das Vulgärlatein. Heidelberg 1913, 36; zum Germanischen Ringe - TAYLOR (Anm. 9) 66. BATTISTI, C. - Alessio, G.: Dizionario etimologico italiano 5. Firenze 1957, 3881 zufolge stammt das italienische Wort aus dem fränkischen Wort und unter italienischen Forschern ist die These des langobardischen Ursprungs aus treuua verbreitet (CortelazZO - Zolli (Anm. 1) 1733 mit Lit.; De MAUrO, T. - MANCINI, M.: Dizonario etimologico. I grandi dizionari Garzanti. Cernusco 2000, 2199), jedoch sind diese Auffassungen lautlich nicht möglich (s. schon BRÜCH, J.: Rezension zu G. Rohlfs, Historische Grammatik der italienischen Sprache und ihre Mundarten I. RomForsch 65 [1954] 436-458, hier 452-453 mit Lit.). Meyer-Lübke behauptet, es sei möglich, dass alle Formen auf das gotische Wort zurückgehen, allerdings können die französischen und katalanischen Formen das gotische Wort nicht fortsetzen.

${ }^{22}$ Zum Lautlichen vgl. RingE-TAYLOR (Anm. 9). Man beachte, dass es keine armenische Entsprechung gibt (contra REY, A. [Hg.]: Dictionnaire historique de la langue française. Paris 2016, 1270), dies ist ein Missverständnis des Hinweises auf das bretonische Wort in ERNOUT-MEILLET (Anm. 1) 628 (,,britt.: arm. leo"). 
Gallien praktisch identisch ist. ${ }^{23}$ Es bleibt daher nur noch die Möglichkeit, dass ein gallisches Wort leuga tatsächlich existierte (für ein weiteres, orthographisch-phonetisches Argument s. noch unten). Dabei handelt es sich allerdings wegen der oben genannten Gründe um eine Entlehnung. Da es gleichzeitig in der Nachbarschaft ein gleichbedeutendes germanisches Wort gab (und zwar nur dort), liegt die Annahme nahe, dass es sich um ein germanisches Lehnwort im Gallischen handelt. Ein solches Szenario ist auch sprachwissenschaftlich plausibel: bekanntlich gibt es viele keltische Lehnwörter im Germanischen, ${ }^{24}$ weshalb germanische Lehnwörter im Keltischen keine Überraschung darstellen. Solche Lehnwörter sind bekannt: zumindest urkelt. *bukko- ,Ziegenbock' < (ur)germ. *bukka- / *bukkan- ,dgl. ${ }^{625}$ und urkelt. *siglo,Segel' < urgerm. *sigla- ,dgl.' ${ }^{26}$

Das Problem besteht letztlich darin, wie die Form von leuga erklärt werden kann, vor allem weil als mögliche Vorstufen westgerm. *leuwa- und goth. *liggwain Frage kommen. Lautsubstitution in *leuwa- mit *g ist nicht motiviert (es gab *w im Gallischen), eine Metathese in *liggwa- kann sogar ausgeschlossen werden, weil sonst ein unregelmäßiger Diphthong *-eu- entstanden wäre. Gegen die gotische Lösung spricht auch die geographische Entfernung: zwar standen die Sprecher des Vorgotischen mit keltischen Sprechern im Kontakt, sie lebten (unabhängig von der bisher nicht eindeutig identifizierten Heimat) allerdings am Rande des keltischen Sprachgebiets (gemeint ist die Region der Unteren Weichsel und die Beziehung zu den Lugiern), ${ }^{27}$ weshalb das Wort praktisch durch ganz Europa hätte wandern müssen, was nicht besonders wahrscheinlich ist. ${ }^{28}$ Da wegen des galatischen Belegs das Wort spätestens im 3. Jh. v. Chr. im Gallischen bekannt war, bleibt auch sprachchronologisch

${ }^{23}$ S. zuletzt ESKA, J. F.: A Salvage Grammar of Galatian. Zeitschrift für celtische Philologie 60 (2013) 51-63, hier 51 mit Lit.

${ }^{24}$ S. z.B. SCHUMACHER, S.: Die Deutschen und die Nachbarstämme: Lexikalische und strukturelle Sprachkontaktphänomene entlang der keltisch-germanischen Übergangszone. Keltische Forschungen 2 (2007) 167-207, hier 172-174.

${ }^{25}$ SCHUMACHER (Anm. 24) 173-176.

${ }^{26}$ SChriJver (Anm. 11) 357 mit Lit., vgl. auch Evans, D. E.: Celts and Germans. Bwlotin y Byrdd Gwybodau Celtaidd / The Bulletin of the Board of Celtic Studies 29 (1980) 230-255, hier 250-251 mit Lit.; SCHMIDT, K. H.: Keltisch und Germanisch. In UnTERMANN, J. - BROGYÁNYI, B. (Hg.): Das Germanische und die Rekonstruktion der Indogermanischen Grundsprache. Akten des Freiburger Kolloquiums der Indogermanischen Gesellschaft, Freiburg, 26.-27. Februar 1981. Amsterdam-Philadelphia 1984, 113-153, hier 123 (und THIER, K.: Language and Technology: Some Examples from Seafaring [Germanic and Celtic]. TPhS 109 [2011] 186-199, hier 189-190, die für eine umgekehrte Entlehnung argumentiert). Weder MATASOVIĆ (Anm. 6) noch seine Rezensentin (BALLES, I.: Rezension zu R. Matasović, Etymological Dictionary of Proto-Celtic. Zeitschrift für celtische Philologie 58 [2011] 265-289, hier 274) ziehen diese Lösung beim ersten Wort in Betracht, das zweite Wort wurde nicht aufgenommen. Nach GRIEPENTROG, W.: Die Wurzelnomina des Germanischen und ihre Vorgeschichte. Innsbruck 1995, 89 mit Lit. stammt gall. *brāca ,Hose' aus phonologischen Gründen nicht aus urgerm. *brōk- ,dgl.', sondern umgekehrt (contra z.B. EVANS [Anm. 26] 251 mit Lit. und SCHMIDT [Anm. 26] 123). S. noch SCHMidT (Anm. 26) 124-125 und SCHUMACHER (Anm. 24) 176 für camisia als weiteres mögliches Beispiel.

${ }^{27}$ GREEN, D. H.: Language and History in the Early Germanic World. Cambridge 1998, 156-158.

${ }^{28}$ Nur der Vollständigkeit halber soll erwähnt werden, dass leuga kein got. Lehnwort im Lateinischen darstellen kann, weil es früher belegt ist als die gotisch-römischen Kontakte (inschriftlich schon 121 n. Chr., ThLL [Anm. 1] 1196). Der galatische Beleg schließt diese Möglichkeit jedenfalls aus. 
äußerst fraglich, ob man schon so früh überhaupt vorgotische Formen annehmen kann. Die westgermanische Form *leuwa-, die ihrerseits mit der urgermanischen Form praktisch identisch ist (abgesehen von der Resyllabifizierung aus *lewwa-, die sich aber auch im Gallischen vollziehen konnte), scheint daher ein besserer Kandidat zu sein.

Ein Problem besteht vor allem darin, dass die Form von leuga nicht so eindeutig ist. Obwohl das Wort in den Inschriften immer als leuga erscheint (falls nicht abgekürzt), wird es von den Autoren auch als leuca (Hier. in Ioel 3.18) und leuva erwähnt. ${ }^{29}$ Die Form leuca passt sehr gut zu einem bekannten aber bislang wenig verstandenen Phänomen im Gallischen: der graphischen Variation zwischen $\langle\mathrm{c}\rangle$ und $\langle\mathrm{g}\rangle$, wobei in der überwiegenden Mehrheit der Fälle $\langle\mathrm{c}\rangle$ für $\langle\mathrm{g}\rangle$ steht (z.B. Argantomagus / arcantodan, aber auch Diviciacus / $\delta \varepsilon 10 v u \gamma(\alpha \gamma o \sigma) .{ }^{30}$ Ebenso ist es hier der Fall: die Ursprünglichkeit des [g] im Falle von leuga ist durch die romanische Fortsetzung, ${ }^{31}$ die Inschriften ${ }^{32}$ sowie die griechische Umschrift von Hesychios gesichert. Die Ursache für diese Variation und die Frage danach, ob sie lautlich oder graphisch bedingt ist, sind bis heute unklar. ${ }^{33}$ Dieses Phänomen ist jedenfalls ein klarer Beweis dafür, dass es sich tatsächlich um ein gallisches Wort handelt, die antiken Autoren Recht hatten und dass die korrekte Form leuga und nicht leuca lautet, wie es von einigen Forschern gebucht wurde. ${ }^{34}$ Das gallische /g/ tendiert allerdings dazu, intervokalisch zu schwinden (das ist die sog. gallische Lenition), weshalb solche Dubletten entstanden, wie Catu(g)enus, Magiona / Maionus, oder Ma(g)iorix. ${ }^{35}$ Während eines Lautwandels und auch danach kommt es oft dazu, dass die Sprecher das Ergebnis des Lautwandels korrigieren und den ursprünglichen Zustand wiederherzustellen versuchen. Da sie allerdings keine Sprachwissenschaftler sind, kommt es dabei oft zu falschen Wiederherstellungen, sog. hyperkorrekten Formen. Im Falle einer Entlehnung ist es einem Sprecher sogar unmöglich zu wissen, wie die frühere Form ausgesehen hat, wenn er die Quellensprache nicht kennt. Einem Gallier, der kein (West)Germanisch konnte (was vor und um die Geburt Christi der Normalfall war), hätte *leuwa ohne weiteres als ,reparationsbedürftige“ Form vorkommen können, weshalb leuga restituiert werden konnte (die frühe Datierung des Wortes wegen des galatischen Belegs ist kein Problem: die Lenition fängt sehr früh an, sie ist schon in den frühesten, mit griechischen Buchstaben geschriebenen, gallischen Texten belegt). Daher könnte

${ }^{29}$ HOLdER (Anm. 1) 198-199; COROMINAS (Anm. 1) 625 mit Diskussion zu den Manuskripten von Isidorus; BILLY (Anm. 14) 94; ADAMS (Anm. 1) 355 Anm. 383.

${ }^{30}$ Für Beispiele s. EvANS (Anm. 11) 400 mit Lit.

${ }^{31}$ Vgl. schon COROMINAS (Anm. 1) 625.

32 BATTISTI-Alessio (Anm. 14) 2193.

${ }^{33}$ Evans (Anm. 11) 400-403 mit Lit., The Contribution of (non-Celtiberian) Continental Celtic to the Reconstruction of the Celtic 'Grundsprache'. In SCHMIDT, K. H. (Hg.): Indogermanisch und Keltisch. Kolloquium der Indogermanischen Gesellschaft am 16. und 17. Februar 1976 in Bonn. Wiesbaden 1977, 66-88, hier 78; MCCONE (Anm. 6) 85; ESKA (Anm. 11) 864.

34 Z.B. DotTin (Anm. 11) 265; HolthAUSEN (Anm. 8) 200; Bolelli (Anm. 14) 50; VON WARTBURG (Anm. 1) 262; PORZIO GERniA (Anm. 1) 111; AdAMS (Anm. 1) 355.

${ }^{35}$ Für Beispiele s. Evans (Anm. 11) 400 mit Lit.; MCCONE (Anm. 6): 85; LAMBert (Anm. 1) 44; ESKA (Anm. 11) 864. 
die Form leuva, die bei einigen Autoren erscheint, statt eines spätlateinischen Lautwandels eher die Normalform des gallischen Wortes zeigen. ${ }^{36}$

\title{
3. ZUSAMMENFASSUNG
}

Die traditionelle Erklärung, dass leuga ein gallisches Lehnwort im Lateinischen ist, das in den romanischen Sprachen weiterlebt und auch ins Angelsächsische entlehnt wurde, lässt drei Probleme ungeklärt: den im Keltischen unüblichen eu-Diphthong, die protoromanische Form *legua, und dass das angelsächsische Wort aus phonologischen Gründen keine Entlehnung aus dem Lateinischen darstellen kann. All diese Probleme können durch ein germanisches Wort *lewwa-gelöst werden, das im Angelsächsischen lautgesetzlich als lēowe fortgesetzt wird, durch dessen gotische Fortsetzung *liggwa die romanischen Formen regelmäßig erklärt werden können und dessen westgermanische Form *leuwa- ins Gallische entlehnt wurde, wo wiederum die Form leuga durch Hyperkorrektion entstand.

\author{
Zsolt Simon \\ Ungarische Akademie der Wissenschaften \\ Forschungsinstitut für Sprachwissenschaft \\ Budapest \\ Ungarn \\ zsltsimon@gmail.com
}

Open Access. This is an open-access article distributed under the terms of the Creative Commons Attribution 4.0 International License (https://creativecommons.org/licenses/by/4.0), which permits unrestricted use, distribution, and reproduction in any medium, provided the original author and source are credited, a link to the CC License is provided, and changes - if any - are indicated. (SID_1)

\footnotetext{
${ }^{36}$ Wäre die Form leuga die Grundlage, könnte man weder die angelsächsische noch die romanischen Formen erklären, weil *leuga- nicht zum urgerm. *lewwa- / urwestgerm. *leuwa- / goth. *liggwaführen kann. Rein theoretisch könnte man auch annehmen, dass das ags. Wort aus *leuwa- und zwar aus der Sprache der in Britannien ankommenden Gallier (für den jüngsten Überblick s. LAMB, A. W.: The Belgae of Gaul and Britain: Revisiting Cross-Channel Contacts in the Later Iron Age. In PAvúK, P. KLONTZA-JaklovÁ, V. - Harding, A. [Hg.]: EY $\triangle$ AIMSN. Studies in Honour of Jan Bouzek. Prague 2018, 335-357) stammt und daher kein Erbwort ist. Occam's Rasiermesser erfordert allerdings, dass ags. lēowe aus einem westgerm. *leuwa- herzuleiten ist, sofern diese westgerm. Form durch die aus den romanischen Formen zu rekonstruierende got. Form vorausgesetzt ist.
} 
